\title{
Development of a virtual management system for the cutting process using neural networks
}

\author{
Irina Ovsyanikova ${ }^{1, *}$, and Aleksandr Tarapanov ${ }^{2}$ \\ ${ }^{1}$ The Orel State University of Economics and Trade, Oktyabrskaya street, Orel, 302028, Russia \\ ${ }^{2}$ Orel State University named after I.S. Turgenev, Komsomolskaya street, 95, Orel, 302026, Russia
}

\begin{abstract}
The article deals with the problems of choosing the neural network configuration for blade processing control. The analysis of input parameters is carried o7ut and the virtual representation of cutting's technological process is simulated. A mathematical control model that allows to mutually transform processes depending on the control criteria is proposed. The configuration of the neural network, that allows to provide the most productive and stable operation with insufficient and contra dictionary input parameters is established.
\end{abstract}

\section{Introduction}

The choice of the neural network configuration for controlling the blade processing is a complex multiargument problem due to nondeterministic, various physical nature phenomena, accompanying the destruction of the billet's surface layer. As a rule, various combinations of theoretical and experimental approaches are used to analyze the cutting process: the positions of the theory of surface engineering, the method of similarity in cutting metals, and so on [1]. They combine the most advantageous qualities of the mentioned directions: the good reliability of the experimental approach and the global generality of the theoretical one. At the same time - the main statements of these studies were related to the parts' shaping for certain operations and transitions.

The method of parameter analysis and control of processing of structural materials [2] allows to virtually transform one way of processing a nominal surface into another way and to make the choice of the most rational one. The optimal machining option can be chosen by such criteria as the number of shaping motions, the configuration of the cross-section of the cut layer, the ability to use several teeth at a time when processing, etc. It's important to take into consideration the process parameters choosing the optimal machining method.

The self-adaptability in the method is achieved by a discrete change in parameters according to the specific features of each machining process. Essentially, the method allows to monitor each and every point in the processing area.

The movement of a plurality of points of the cutting blades in accordance with the principles of shaping and in accordance with the features of the movement in the direction of the main motion and the movements of the feeds for discrete time intervals.

\footnotetext{
*Corresponding author: ovsynikova2012@yandex.ru
} 


\section{Theoretical part}

To analyze the geometric parameters of the cutting processes, it is necessary to divide the shaping motion of the tool's cutting blade into the main cutting motion and the feed motion. As a result we obtain the following independent parameters:

$\varphi(V)$ - main cutting motion parameter;

$\varphi\left(S_{n}\right)$ - summed movement federate parameter;

$\varphi(l)$ - cutting blade parameter.

The fact that each point of the researched space in the processing area simultaneously possesses the qualities of the machined billet and the tool, is determined by its function-spatial purpose and time, includes the possibility of control within the system according to the outer given parameters and limitations, provides multivariance to the cutting control and analysis system. In reference to them we can name qualitative indicators of the nominal surface, cutting modes, parameters of tool installation, processing time and its cost.

In the generalized mathematical representation of the cutting scheme, which represents the dependence of the coordinates of the points of the cutting blades on independent parameters, the time $\tau$ and the control parameter $u$ are entered:

$$
\begin{aligned}
& x=f\left(\varphi(V), \varphi\left(S_{n}\right), \varphi(l), \tau, u\right), \\
& y=f\left(\varphi(V), \varphi\left(S_{n}\right), \varphi(l), \tau, u\right), \\
& z=f\left(\varphi(V), \varphi\left(S_{n}\right), \varphi(l), \tau, u\right) .
\end{aligned}
$$

The control parameter, in its turn, can be a function of external given parameters.

Mathematical analysis of the mapping of the cutting scheme allows to determine the kinematic changes in the back and front corners, the angle of inclination of the cutting edge, the thickness of the cut layer for any point of the profile and at any time [1]. This allows to determine velocity vectors of movement in the direction of feed and movement of cutting.

The machining option is chosen to optimize the shaping motions, the configuration and size of the cross-section of the shear layer, the possibility of using several teeth at the same time. When choosing the method of processing, the parameters of the technological process and the limitations in accuracy, roughness of the nominal surface, the prime cost of processing exert an influence significantly.

Tasks with a high degree of uncertainty are very often solved in the technology of engineering. In the solution space, a set of solutions with a different probability density is obtained. To solve such tasks like these, researchers use the theory of fuzzy sets, heuristic methods for selecting parameters using priorities of importance, values of preference functions, etc. One of the main methods of working with uncertainties is the Bayesian probabilistic approach.

The action of the cutting force during the processing of the nominal surface causes bending dynamic deformations of the workpiece, flexural deformation of the mandrel, and also elastic depressions of the workpiece and mandrel, due to the finite stiffness of the supports.

When machining with a multilevel tool, the action of cutting forces from all the teeth of the milling cutter in the work may happen as well. As the cutting force is the sum of the repeated impulses, the deformation of the system can be represented as a sum of deformations caused by isolated impulses shifted in phase relatively to the other.

The initial data for constructing a dynamic system that provides the possibility of changing the results obtained earlier are given below:

- specified parameters of accuracy, roughness of the machined surface and tool life; 
- properties of the material that is being processed;

- properties of the material of the cutting part of the tool;

- parameters of the process system;

- method of fixing the workpiece;

- dimensions and parameters of the helical surface;

- tool dimensions and geometry of its cutting part and parameters of the cutting process.

During the data preparation for the simulation of the virtual workflow, it's necessary to perform the following operations:

- interpretation of data - choosing a solution from a fixed set of alternatives based on the entered information about the current situation.

- diagnosis - identifying the causes that led to the emergence of the situation.

- correction - diagnosis, supplemented with the possibility of evaluation and recommendations for corrective actions for deviations from the normal state of the situations in question.

For the details' surface blading, the problem of recognition of situations is solved, the belonging of surfaces to a certain class from a given set is defined, input parameters and input conditions are determined. And among the set of solutions we find the only possible solution satisfying all the constraints and final goal.

Creating a management model allows to form a technological environment and virtually manage it, avoiding the influence of random factors, that take place in the practical experiment. To calculate probabilistic estimates, special parameters of "deregulation" are introduced into the model. The main task of information modeling is to provide the required output values of the parameters of the technological process, and the indirect task is to minimize the influence of random factors.

The evidence that for any algorithm there exists a neural network (NS) realizing it is given in the literature $[3-10]$. The NC allows to work with noisy data and adapt to any external influences and simulate various nonlinear processes.

The control system model is built on the basis of fully connected NCs, due to the fact that the same number of neurons NCs have a greater number of inter-neural connections, and this increases the information capacity of the network. Full-bodied architecture is considered to be the most universal one. The adaptive control HCs are designed in such a way that the output of the controlled model corresponds to the output of a predetermined model that has the required characteristics.

The training of the virtual management system is based on the principle of training, consisting in the gradual accumulation of experience in the formation of the behavior of the system with a high degree of uncertainty of its initial states. As a result, the system functions better. All the ways of accumulating experience have a common feature, namely, the gradual separation of the field of knowledge from the whole set of ignorance. In the theory of learning systems, this feature is reflected in the creation of automatic classification systems or pattern recognition.

In any recognition algorithm, there is always a procedure for comparing the input signal with the available standards in memory. After determining the overall structure of the network, it is necessary to clarify its parameters. The network parameters include the number of input and output neurons, the number of neurons in the Kohonen and Grossberg layers, and the magnitude of the synaptic connections.

To solve this problem, counter propagation network (CPN) that consists of two layers of neurons - the Kohonen layer and the Grossberg layer, can be used. It works with binary vectors consisting of zeros and ones, or with continuous vector. As a result of learning process, input vectors are associated with output ones and, when the network is trained, the feed of the input images leads to getting output ones. The correct output can be obtained also when the input is incomplete or somewhat incorrect. 
Computations with higher speeds are provided by the parallel configuration of the HC. For approximation of nonlinear representations with the necessary degree of accuracy, the networks containing nonlinear components are used.

Learning the algorithm of inverse propagation of error assumes two passes on all the network's layers: direct and reverse. At a direct pass the input vector is fed to the input layer of the neural network, and after that spreads on a network from layer to layer. As a result, a set of output signals, which is the actual response of the network to this input image, is generated.

As a result, each combination of all input signals that change in some given ranges will have its own output signal value.

Currently, a number of different architectures for building neural network control systems have been developed and widely used. The neural network controller performs the development of an adequate control signal for controlling the dynamics of the controlled object's states. States change happens according to the optimal trajectory.

In the process of learning, an automatic construction of generalizing rules or functions that describe the belonging of situations to classes that the system will later use when interpreting unfamiliar situations is carried out. From the generalizing rules, the knowledge base is automatically formed, which is being periodically adjusted as information about the analyzed situations accumulates.

If the network is trained well, it acquires the ability to model an unknown function that connects the values of input and output variables, and finally, such a network can be used for predictions in situations when the output values are unknown.

The adaptation of typical development to features of a problem area is carried out at typical designing. To implement this approach, certain tools are used for component (assembly) design of information systems (R / 3, BAAN IV, Prodis, etc.).

The structural synthesis of complex dynamic process control systems in a combination of concepts, methods and mathematical apparatus of modern nonlinear control theory with the theory of trained artificial neural networks, opens a wide range of opportunities for improving the quality of machined surfaces with a cutting tool.

\section{Conclusion}

1. It would be appropriate to choose configurations of neural network to control blade processing through the complex method of analysis and virtual presentation.

2. The technique of research and management of blading processing allows to transform virtual processes represented by mathematical mappings that provide a wide variation of the properties of the machined parts, parameters of процессов обработки and parameters of the cutting tool.

3. The counterpropagating neural network, characterized by rapid training and stable operation with incomplete or incorrect input parameters is suggested to control the blading process and a wide variation of the input signal spectra.

\section{References}

1. A.N. Afonin, Yu.S. Stepanov, A.V. Kirichek, A.S. Tarapanov. Scientific and pedagogical preparation of Master of Engineering and technology: Textbook for high schools (Moscow: Mechanical Engineering, 2009) 
2. G.A. Kharlamov, A.S. Tarapanov, V.S. Shorkin Mathematical foundations of virtual representation and analysis of blade processing Publishing house "Mechanical Engineering". (Directory. Engineering Journal vol 2 - 3, 2003).

3. B.M. Brzhozovsky, M.B. Brovkova, V.V. Martynov Nonlinear Dynamics and applied synergetics: Materials of Intern. Conf. - Komsomolsk-on-Amur, (2003)

4. L.N. Yasnitsky Introduction to Artificial Intelligence Textbook for high school students 2 nd ed., Rev. (Moscow: Publishing Center "Academy", 2008)

5. T. Kohonen Self-organization and associative memory. (Series in Information Sciences, vol. 8. Berlin: Springer Verlag. 1984)

6. Haykin Simon. Neural networks. Full course 2 nd ed., Rev.: translated from English. (M .: OOO "I. D. Williams ", 2006)

7. R. Dorf, R. Bishop Modern management systems (Moscow: Laboratory of Basic Knowledge, 2009)

8. A. Galushkin, Neural networks. Fundamentals of the theory (Hot Line-Telecom, 2012)

9. I.V. Ovsyanikova, A.S. Tarapanov, V.S. Shorkin Control parameters of the process of complex surface formation. (Fundamental and Applied Problems of Technics and technology, 2, pp. 56-60. 2012)

10.I.V. Ovsyanikova, E.A. Sychev, A.S. Tarapanov Information modeling of adaptive control of EDM (Fundamental and Applied Problems of Technics and technology, 5, pp. 103-107. 2013)

11. Russell, Stuart J. \& Norvig, and Peter Artificial Intelligence: A Modern Approach (second ed.), Upper Saddle River (New Jersey: Prentice Hall, 2003)

12. Tadeusewicz Ryszard, Borovik Barbara, Gonchazh Tomasz, Lepper Bartosh. An elementary introduction to the technology of neural networks with examples of programs. (Moscow: Hot line - Telecom, 2011)

13. Rutkowski Leszek Methods and technologies of artificial intelligence. Rudinsky Publishing House: (Hot Line - Telecom, 2010) 